\title{
La formación musical del profesorado especialista en los CEIP gallegos
}

\author{
Rocío Chao Fernándezi \& Mª Dorinda Mato Vázquezii \\ Universidade de A Coruña, Espanha \\ Vicente López Pena \\ Universidade de Cádiz, Espanha
}

\begin{abstract}
Resumo
La formación del profesorado es una de las preocupaciones de los países que encabezan los resultados del Informe PISA, pues la titulación del personal docente no supone confirmar su eficacia como profesionales ni la calidad de su trabajo. La escasa formación musical de los discentes que acceden en los últimos años a diversas Facultades de Educación de Galicia nos ha inducido a analizar las causas y consecuentemente averiguar cuál es la formación del profesorado encargado de la enseñanza musical en los centros de Educación Primaria (CEIP). Se ha aplicado un cuestionario, con una muestra aceptante de 124 docentes, de los cuales 112 pertenecen a centros públicos y 12 a concertados. Los resultados pueden ayudar a los profesionales a mejorar y abordar de modo diferente su acción educativa.
\end{abstract}

Palabras-clave

Formación del profesorado; Educación Musical; Educación Primaria; Galicia

\section{Introducción}

La formación del profesorado - pieza clave de una educación de calidad - es una cuestión que, asiduamente, forma parte del debate en el ámbito educativo y, en este período de cambios curriculares, dicha discusión 
se ve fuertemente reavivada. Evidentemente, para mejorar cualquier sistema educativo es fundamental realizar una formación inicial y permanente de sus docentes, así como una continua motivación e incentivación. Por tanto, como sostiene Imbernón (2012), la investigación se divisa como uno de los procesos importantes en el perfeccionamiento de los profesores. Sin embargo, a pesar de esa necesidad que evidencian numerosos expertos, el informe realizado por los miembros de la comisión de investigación de la International Society for Music Education (en López de la Calle, 2009) revela poco interés en acometer estudios en este ámbito. También, la consultora estadounidense McKinsey and Company en su informe (Mourshed, Chijioke, \& Barber, 2010) asegura que el éxito de un sistema educativo competitivo está en elegir a los mejores alumnos para convertirlos en profesores.Así mismo, los mejor calificados en los informes PISA (2012, 2013), realizados por la Organización para la Cooperación y Desarrollo Económico (OCDE) desde el año 2003, concuerdan en que efectúan una exhaustiva selección de sus futuros profesores entre los estudiantes con mejor expediente. Además realizan una rigurosa entrevista donde los candidatos deben demostrar sus competencias emocionales y pedagógicas. En la misma línea se encuentran Zabalza \& Zabalza (2011), con quien coincidimos cuando afirman que garantizar la titulación del personal no supone confirmar su eficacia como profesionales ni la calidad de su trabajo. No obstante, en los sistemas muy burocráticos, como el nuestro, se tiende a dar excesiva importancia a los títulos y poca a las competencias reales de sus poseedores.

Ciertamente, el docente debe poseer los conocimientos necesarios para llevar a cabo todas las tareas relacionadas con el diseño, la programación y puesta en práctica del proceso de enseñanza (Márquez, 2009). Consideramos que a estos conocimientos es necesario añadirles una formación pedagógica que garantice una correcta transmisión de los mismos, abogando por el paradigma del profesor como investigador y profesional reflexivo; educador capaz de despertar el interés por aprender, cómo aprender y mantener al día estos conocimientos (Galvis, 2007). Así pues, creemos que la investigación en torno al conocimiento profesional del futuro profesor debe concebirse como parte de su desarrollo profesional, y como precisan Climent y Carrillo $(2002,2003)$ es, a su vez, fuente de información para organizar la formación inicial. Por todo lo expuesto, nuestra preocupación como formadores de profesores está justificada. 
Por otra parte, la importancia que posee la Educación Musical en la formación de las personas es innegable, lo que justifica su inclusión en el ámbito escolar. A pesar de que no siempre se han encontrado los mecanismos adecuados para una puesta en práctica enriquecedora, apoyamos la idea de Touriñán \& Longueira (2010) cuando declaran que la enseñanza de la Música ha evolucionado en las últimas décadas en nuestro sistema educativo, ya que en España esta disciplina no estaba incluida en el currículo escolar. Es a partir de la entrada en vigor de la Ley Orgánica General del Sistema Educativo (LOGSE) en 1990 cuando comienza a recibir la consideración debida (Díaz, 2012), lo que provoca un giro copernicano en la concepción curricular de la Educación Primaria. Desde entonces, la enseñanza de la música ha ido sufriendo vaivenes con las diferentes reformas educativas (Ley Orgánica 10/2002, de 23 de diciembre, de Calidad de la Educación - LOCE; Ley Orgánica 2/2006, de 3 de mayo, de Educación - LOE; y Ley Orgánica 8/2013, de 9 de diciembre, para la Mejora de la Calidad Educativa - LOMCE), en las que nunca ha llegado a ocupar el mismo estatus que otras asignaturas. Nos referimos a las disciplinas instrumentales, especialmente Lengua y Matemáticas. Además, muchos conceptos de estas disciplinas se pueden desarrollar de forma realmente significativa a través de una Educación Musical activa y participativa (Elliot, 2005; Pascual, 2006; Pérez \& Leganés, 2012; Toscano \& Fonseca, 2012).

La reforma de 1990 no solo repercutió en el currículum de Educación Primaria, sino también en la organización docente: en primer lugar porque gran parte del profesorado resultó suprimido y en segundo lugar porque fue necesario dotar a los centros educativos de profesionales especializados en las nuevas disciplinas. Para resolver la situación en numerosas comunidades autonómicas, entre las que se encuentra la gallega, se ofertaron cursos de postgrado a través de los cuales se habilitó al personal para impartir las nuevas especialidades LOGSE, pese a existir dos Universidades en las que se ofrecía la titulación de Maestro con especialidad en Educación Musical (Chao, 2010; Chao, Mato, \& Muñoz, 2014). Al respecto, Camacho (2006) afirma que a esta nueva situación debieron adaptarse aquellos profesores que en su clase explicaban contenidos tan variados como Trigonometría o la Primera Revolución Industrial, sin tener en cuenta sus sentimientos y preocupaciones (Reyes \& Pech, 2007). 
No obstante, para poder extraer el mayor partido posible de cualquier materia es necesario contar con profesionales perfectamente cualificados (Márquez, 2009), máxime si se trata de disciplinas cuyos beneficios pueden repercutir no solo en el rendimiento académico de los alumnos, sino también en su comportamiento (Levitin, 2011) y en su integración social (Bernabé, 2012) al relacionarse a través del canto y formación instrumental colectivos.

Así pues, ante la escasez de investigaciones sobre la formación del profesorado encargado de la Enseñanza Musical del alumnado de Educación Primaria, y a la vista de la insuficiente formación de los discentes que acceden a las Facultades de Educación, se lleva a cabo este estudio, que revela la necesidad de fomentar los trabajos de investigación en esta área.

\section{La importancia de la Educación Musical en la formación integral}

Desde la antigüedad se conocen los beneficios educativos de la Música, pero es a partir del siglo XX cuando se realizan investigaciones rigurosas que suscitan numerosas aportaciones a la Pedagogía Musical y que han dado un giro a la forma de entender la didáctica de la misma (Chao, Mato, \& Ferreiro, 2014). La literatura educativa musical ha presentado, en los últimos años, importantes investigaciones de reconocidos y prestigiosos autores como Dalcroze, Hemsy de Gainza, Swanwick, Tomatis, Vallés y Willems, entre otros (Brufal, 2013). Todos ellos dedicaron parte de su trabajo a buscar respuestas innovadoras o diversas formas de mejorar la enseñanzaaprendizaje a través de la Música. En esta línea, Zoltán Kodály, en Chao (2010), desarrolló uno de los primeros estudios relevantes en los que indicó los ámbitos en los que influye positivamente el aprendizaje musical. Continúan y completan el estudio Levitin y Álvarez (2008) y Levitin (2011), demostrando que si se trabaja adecuadamente esta disciplina, el cerebro produce un aprendizaje acelerado y significativo, a la vez que facilita la sociabilización entre individuos y modifica el estado de ánimo.

Asimismo Pérez y Leganés (2012) muestran que la experiencia musical activa la imaginación y la creatividad, y construye el fundamento desde el cual actúan los procesos de cognición, percepción, atención, memoria, inteligencia, pensamiento y lenguaje. Al respecto, Pascual (2006) 
asegura que a partir de la Música se pueden desarrollar todos los objetivos, contenidos y actividades del proceso de enseñanza-aprendizaje que tienen lugar en la escuela. Es por eso que adquiere gran importancia el papel que adopta el docente, es decir, lo importante es cómo se lleva a cabo el proceso educativo (Díaz, 2012).

Márquez, en un estudio realizado a profesores en el año 2009, comprobó que todos los entrevistados manifestaron carencias en su formación inicial, especialmente el hecho de que no se les formara para la labor que iban a tener que desempeñar. Así pues, ante la repercusión que, sin duda, tiene una correcta Educación Musical en la formación integral de los discentes, consideramos que es primordial que el profesorado encargado de su transmisión debe estar correctamente cualificado, y poseer no sólo un sólido conocimiento de la materia, sino también las adecuadas estrategias pedagógicas.

\section{Objetivos}

El objetivo nuclear de esta investigación es conocer la forma de acceso y preparación del profesorado encargado de impartir Educación Musical en los CEIP de Galicia y las fuentes de su formación; así como indagar cuál o cuáles son las metodologías mayoritariamente empleadas en el quehacer diario.

\section{Metodología}

Nuestro interés por dotar a nuestra investigación del debido rigor científico nos impulsó a decantarnos por llevarla a cabo a través de la aplicación de un cuestionario a los profesores encargados de impartir la asignatura de Música en todos los centros de Primaria de la Comunidad Gallega, además de entrevistas semiestructuradas. Tras la recogida de información hemos procedido al análisis individual de los datos extractados.

\section{Muestra}

Hemos realizado a través del Diario Oficial de Galicia (D.O.G.A.) un estudio de los centros de Educación Primaria existentes en la Comunidad Gallega, tras lo cual se ha realizado una selección de forma aleatoria de 400 
Colegios, de los cuales 320 eran públicos, 60 concertados y 20 privados. La muestra aceptante fue de 124 centros de Educación Primaria, de los cuales 112 son públicos (90\%) y 12 concertados (10\%). De los privados no respondió ninguno. En total constituyen el $31 \%$ de la muestra invitada, según se aprecia en el gráfico 1.

\section{Gráfico 1. Muestra aceptante}

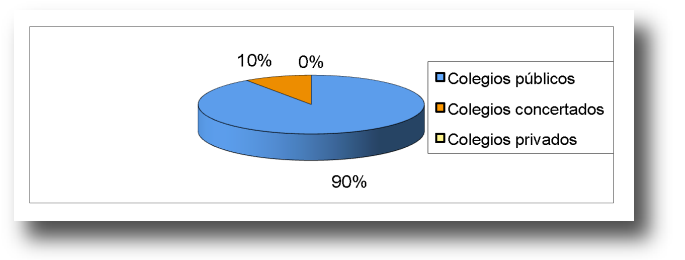

En cuanto a la distribución por provincias, podemos comprobar en la tabla 1 que existe una gran diferencia entre el número de centros de A Coruña y Pontevedra en comparación con Lugo y Orense. Lo mismo sucede con el total de cuestionarios que han contestado. Así, en A Coruña, a pesar de haber 333 centros, sólo hemos recibido 44 encuestas cumplimentadas, lo que supone un porcentaje del $13,21 \%$. En Lugo el número de centros es de 122 y las respuestas han sido de 29 (23,77\% del profesorado objeto de estudio). En Orense hay 105 centros y respondieron 19, con lo que la muestra es de un $18,89 \%$. Con respecto a Pontevedra, descubrimos que existen 263 centros, de los que tan sólo hemos recibido 32 cuestionarios, lo que supone un $12.17 \%$ de la totalidad de la población del estudio en cuestión.

Tabla 1 - Distribución de la muestra por provincias

\begin{tabular}{|cccc|}
\hline Provincias & Centros & Muestra Aceptante & Total porcentual \\
A Coruña & 333 & 44 & $13,21 \%$ \\
Lugo & 122 & 29 & $23,77 \%$ \\
Orense & 105 & 19 & $18,89 \%$ \\
Pontevedra & 263 & 32 & $12,17 \%$ \\
TOTAL & 823 & 124 & $15,06 \%$ \\
\hline
\end{tabular}




\section{Resultados}

La primera parte del cuestionario hace referencia a los aspectos personales del profesorado: género, edad, ciclos educativos en los que trabaja, titulaciones, plan de estudios, especialidades y experiencia docente. La segunda parte trata de analizar los aspectos concretos de la práctica docente en la clase de Música: metodologías utilizadas, adquisición de conocimientos y su valoración sobre los contenidos de la Educación Musical.

En relación al género, podemos constatar que han participado 72 mujeres y 52 hombres, lo que nos lleva a considerar que existe un cierto equilibrio en cuanto al sexo de los profesores de Música de la muestra, aunque se inclina ligeramente hacia el lado femenino (Gráfico 2).

Gráfico 2 - Sexo

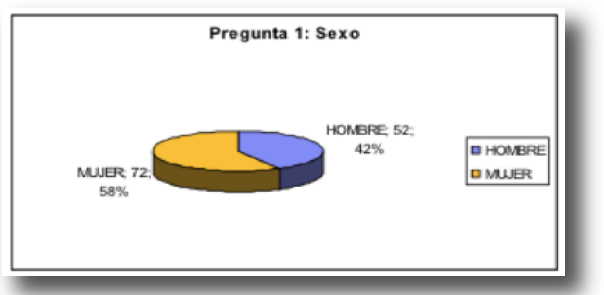

En cuanto a la edad media de los docentes, se circunscribe, fundamentalmente, entre 41 y 50 años (Tabla 2), lo que denota un profesorado de mediana edad, representando porcentualmente el $48.39 \%$ de la muestra.

Tabla 2 - Edad del profesorado

\begin{tabular}{|ccc|}
\hline Edad & Muestra & Porcentaje \\
Entre 20-25 años & 2 & $1,6 \%$ \\
Entre 26-30 años & 24 & $19,36 \%$ \\
Entre 31-35 años & 10 & $8,07 \%$ \\
Entre 36-40 años & 17 & $13,71 \%$ \\
Entre 41-45 años & $\mathbf{2 2}$ & $\mathbf{1 7 , 7 4 \%}$ \\
Entre 46-50 años & $\mathbf{3 8}$ & $\mathbf{3 0 , 6 5 \%}$ \\
Entre 51-55 años & 7 & $5,64 \%$ \\
Más de 56 años & 4 & $3,22 \%$ \\
TOTAL & 124 & $100 \%$ \\
\hline
\end{tabular}


La variable relativa a los Ciclos Educativos en los que imparten la docencia comprende 4 opciones: $1^{\circ}, 2^{\circ}, 3^{\circ}$ de Educación Primaria y "otros", en los que se podrían localizar respuestas tan extremas como las referidas a $1^{\circ}$ ciclo de Enseñanza Secundaria o a Educación Infantil. A la primera opción han respondido afirmativamente 119 profesores (el $95,97 \%$ ), mientras que a las dos siguientes $\left(2^{\circ}\right.$ y $\left.3^{\circ}\right)$ lo han hecho 120 , lo que representa el $96,77 \%$ de los docentes. En la cuarta, 62 personas declaran impartir Música en Educación Infantil, lo que supone el 50\% del profesorado, asegurando todos ellos que lo hacen por voluntad propia (Tabla 3).

Tabla 3 - Ciclos en los que imparte docencia

\begin{tabular}{|lcc|}
\hline Ciclos & Muestra & Porcentaje \\
$1^{\circ}$ Ciclo & 119 & $95,97 \%$ \\
$2^{\circ}$ Ciclo & 120 & $96,77 \%$ \\
$3^{\text {a } \text { Ciclo }}$ & 120 & $96,77 \%$ \\
Ed. Infantil & 62 & $50 \%$ \\
\hline
\end{tabular}

En cuanto al análisis de las titulaciones, observamos lo siguiente: el 93,55\% declara poseer estudios universitarios de primer ciclo, mientras que el $6,45 \%$ no contesta. Es necesario aclarar, ante esta respuesta, que, pese a que en la actualidad es condición indispensable para opositar al cuerpo de maestros estar en posesión del Grado de Educación Infantil o Primaria, o de la extinguida Diplomatura en Profesorado de Educación General Básica, hasta el año 1987 podía acceder por oposición cualquier licenciado. Además, 12 profesores confiesan tener también estudios de $2^{\circ}$ ciclo, lo que supone el $9,68 \%$ de la muestra. Al ser una pregunta cerrada, no especifican de qué carrera se trata, pero sí explican que es una Licenciatura de Universidad, no de Conservatorio. En el apartado "otros", contestan afirmativamente 23 profesores (Gráfico 3).

Otro dato a meditar es que la mayoría de los encuestados $(58,06 \%)$ procede del plan de estudios de 1971 y el 25.01\% (31 personas) al de 1991. De estos últimos sólo once personas $(8,87 \%)$ aseguran haber realizado la especialidad de Educación Musical. El 14,51\% restante procede del plan de 1967 y anteriores a este. No responden tres personas, lo que representa un $2,42 \%$ (Tabla 4 ). 
Gráfico 3 - Estudios del profesorado

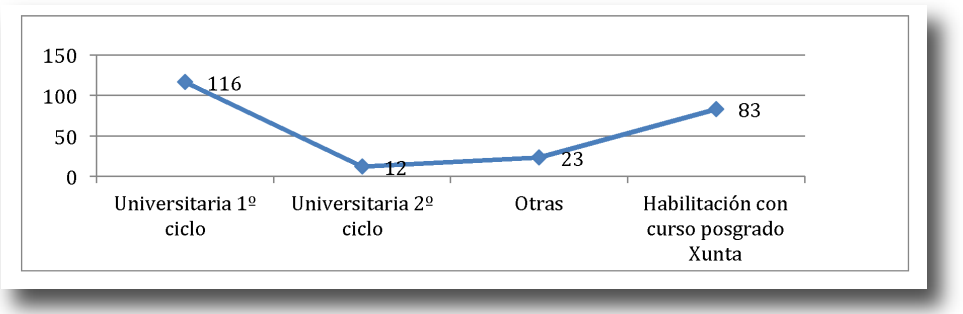

Tabla 4 - Plan de estudios a que pertenece

\begin{tabular}{|lcc|}
\hline Plan de estudios & Muestra & Porcentaje \\
Plan de estudios anterior & 5 & $4,03 \%$ \\
Plan de 1967 & 13 & $10,47 \%$ \\
Plan de 1971 & 72 & $58,06 \%$ \\
Plan de 1991 & 31 & $25,01 \%$ \\
No contesta & 3 & $2,42 \%$ \\
TOTAL & 124 & $100 \%$ \\
\hline
\end{tabular}

La justificación de que 72 profesionales declaren haber cursado el plan de estudios de 1971 puede radicar en el hecho de que se trata de un plan con una gran vigencia (20 años, con la revisión que sufrió en 1977). Como podemos apreciar (Tabla 5), ninguno de los encuestados ha realizado la especialidad de Educación Musical, ya que no existía y se incorporó paulatinamente a los planes de estudio a partir de 1991.

Estimamos necesario clarificar la situación actual: con el Espacio Europeo de Educación Superior desapareció la especialidad de Educación Musical, por lo que el escenario empeoró nuevamente; bien es cierto que algunas Facultades ofertan optativas de Música con el fin de obtener una "mención" relacionada con dicha especialidad; sin embargo, supone, por una 
parte, un retroceso en cuanto a la formación docente y, por otra, no la ofertan todas las Facultades, lo que equivale a una evidente discriminación. En el momento en que se realizó el estudio en Galicia, todavía no se había graduado nadie.

Tabla 5 - Especialidad cursada en el plan de estudios de 1971

\begin{tabular}{|lcc|}
\hline Plan de estudios 1971 & Muestra & Porcentaje \\
Ciencias & 22 & $17,74 \%$ \\
Ciencias Humanas & 5 & $4,03 \%$ \\
Filología & 4 & $3,23 \%$ \\
No contesta & 41 & $33,06 \%$ \\
TOTAL 1971 & 72 & $58,06 \%$ \\
TOTAL MUESTRA & 124 & $100 \%$ \\
\hline
\end{tabular}

Las opciones de acceso para impartir docencia de Música son dos: opositar o conseguir una habilitación. De estas, las primeras se consiguieron con un único año de solfeo cursado en conservatorio. Hemos de subrayar que el $100 \%$ de los encuestados que pertenecen al plan de estudios de 1971 declara haber accedido a impartir clases de Música a través del curso de especialización promovido por la Xunta de Galicia, para dar salida a un elevado número de profesores que veían suprimida su plaza con la implantación de la LOGSE. Otro apunte que debemos hacer notar es que los profesores que pertenecen al plan de estudios de 1991 (Tabla 6) representan solo el $25.01 \%$ de la muestra, lo que semeja un porcentaje pequeño. Además, es sorprendente que, a pesar de que en este plan se contempla la figura del especialista de Música, sólo 11 de las 31 personas que pertenecen a este plan han cursado dicha especialidad (lo que supone el $8.87 \%$ de la muestra total).

Es por eso que consideramos que estos datos son muy significativos, puesto que existen pocos profesionales que hayan realizado estudios específicos para impartir esta materia. Por tanto, la mayoría han sido habilitados, bien a través de cursos de postgrado (con pocos contenidos), bien a través de oposición (los menos). 
Tabla 6 - Especialidad cursada en el plan de estudios de 1991

\begin{tabular}{|lcc|}
\hline Plan de estudios 1991 & Muestra & Porcentaje \\
Infantil & 8 & $6,46 \%$ \\
Primaria & 11 & $8,87 \%$ \\
Música & 11 & $8,87 \%$ \\
Ed. Física & 1 & $0,81 \%$ \\
TOTAL 1991 & 31 & $25,01 \%$ \\
TOTAL MUESTRA & 124 & $100 \%$ \\
\hline
\end{tabular}

En definitiva, podemos concluir que de la muestra de 124 profesores, 113 (el $91.13 \%$ ) no han estudiado la especialidad de Educación Musical y sólo 11 (8.87\%) proceden de estudios especializados en formar profesores para impartir esta disciplina (Gráfico 4).

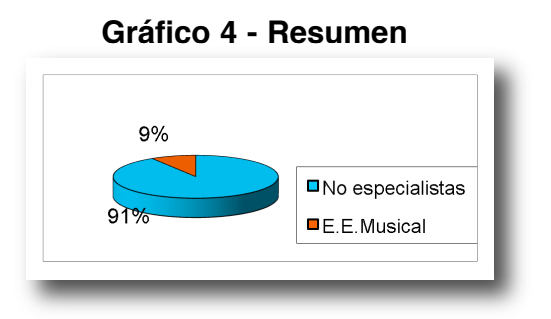

En cuanto a la experiencia docente, observamos que el 39,52\% (49 personas) tiene una experiencia docente entre 21 y 30 años; el 18,55\% (23 personas) entre 11 y 15; el 13,71\% (17) trabajó entre 1 y 5 años; el 12,90\% (16 profesores) entre 6 y 10 ; el 9,68\% (12) entre 16 y 20 ; y el 5,64\% (7 profesionales) ha trabajado más de 31 años (Gráfico 5).

Viene a colación en este punto la opinión de Marcelo $(2009,2011)$, al considerar la formación docente como un proceso amplio y flexible, continuo, sistemático y organizado. En este sentido, la mayor parte del profesorado lleva tiempo suficiente en la docencia para ser considerado experto. Sin embargo, al $91,13 \%$ de la muestra de nuestro estudio no podemos 
concederles este reconocimiento en Educación Musical, porque su experiencia corresponde a otras disciplinas; pues ellos carecen de formación inicial y permanente, además de conocimientos, destrezas y actitudes adecuadas para llevar a cabo una educación de calidad en esta materia.

\section{Gráfico 5 - Experiencia docente}

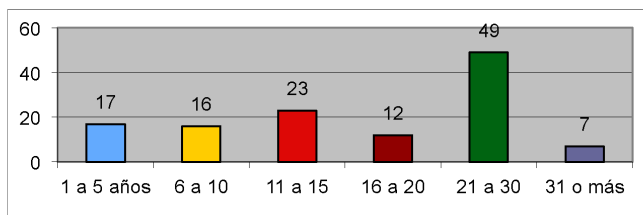

En las entrevistas también hemos preguntado cómo accedieron a dicha especialidad. La respuesta, de nuevo, es contundente: el 94,12\% ha declarado acceder a través del curso de postgrado de la Xunta. Por lo que, este cambio drástico en la trayectoria profesional de estos docentes, que se vieron obligados a habilitarse en una disciplina totalmente ajena a su formación, está provocado fundamentalmente por dos motivos, según los datos extraídos en las encuestas: en primer lugar, por el descenso de natalidad que ha experimentado nuestra Comunidad; en segundo lugar, especialmente por la entrada en vigor de la LOGSE, ya que en su momento supuso una considerable supresión de plazas, que afectó al profesorado de Educación Primaria. Los cursos llamados $7^{\circ}$ y $8^{\circ}$ de Educación General Básica cuyas plazas ocupaban los maestros pasaron a ser $1^{\circ}$ y $2^{\circ}$ de la Educación Secundaria Obligatoria, y ocupados por licenciados en su mayoría.

En otro orden de cosas, ambicionábamos averiguar qué tipo de modelos pedagógicos son los más utilizados por los especialistas de Música en su labor docente y, en consecuencia, la influencia que ello puede tener en la Educación Musical. Debemos precisar que el especialista en esta disciplina debe conocer distintas metodologías para elegir el aspecto que más le guste de cada una. Ahora bien, como expone Brufal (2013), "conocer" una metodología significa haberla vivido uno mismo y tener una serie de criterios 
que nos permitan usar con espíritu crítico, cuidando la unidad y la coherencia de la actividad docente, de manera razonable y eficiente, según nuestra experiencia profesional, los diferentes recursos en las situaciones adecuadas.

Nuestro planteamiento inicial partía de 6 opciones (Dalcroze, Orff, Kodaly, Ward, Martenot y Willems), aunque hemos brindado una última en la que se dejaba libertad a otras aportaciones por parte de los entrevistados. Esta selección está fundamentada en el hecho de que todos ellos son referentes en la Pedagogía Musical, con determinados aspectos comunes y otros que se complementan. Por ejemplo, Dalcroze (1865-1950) sostenía que el ritmo musical debía ser interiorizado por medio del movimiento corporal. En cambio, para Orff (1895-1982) cobraba importancia el descubrimiento del valor rítmico y expresivo del lenguaje hablado y su relación con el lenguaje musical, así como la improvisación instrumental. Kodály (1882-1967) se centró en la importancia del folclore y del canto como medio cardinal para la Enseñanza Musical. Por su parte, a Justine Ward (1879-1975) le debemos revelar y ensalzar los aspectos vocales y expresivos, así como la plasticidad rítmico-melódica. Martenot (1898-1980) se basó especialmente en el desarrollo de la audición interna, aunque también concedió gran importancia a la respiración y a la relajación corporal. Finalmente, Willems (1890-1978) se centró en las relaciones psicológicas establecidas entre la Música y el ser humano, así como en el desarrollo de la audición interior, tomando como punto de partida la Música en sí misma ante cualquier proceso de enseñanzaaprendizaje.

Teniendo esto en cuenta, observamos que los modelos más utilizados por el profesorado son los siguientes: en primer lugar el método Orff, con 113 contestaciones, lo que supone que lo emplea el $91,13 \%$ del profesorado; le sigue el método Kodály, con 86 respuestas, lo que equivale al $69,35 \%$ de los encuestados; el siguiente es el método Dalcroze, con 56 personas $(45,16 \%)$; y le sigue el método Willems, con 52 contestaciones (41,94\%). También son métodos tratados el Ward (3 contestaciones, 2,42\% del profesorado) y el Martenot (con 1 contestación, 0,81\%). Además, 14 personas utilizan "otros" métodos, lo que supone un $11,29 \%$ del total de la muestra (Gráfico 6). 
Gráfico 6 - Metodologías más utilizadas

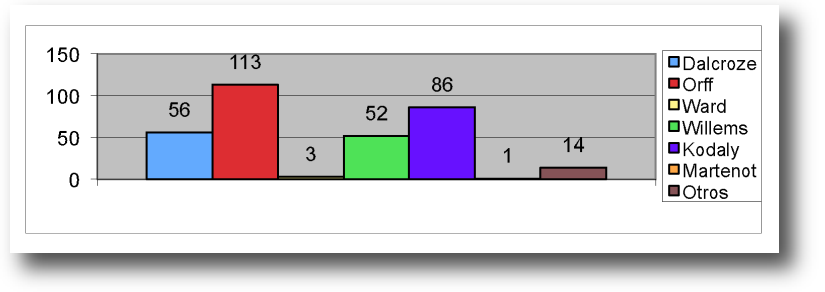

Uno de los entrevistados incluye el método Wuytack en el apartado "otro tipo de metodología", y otro Musicoterapia, lo que supone el $0,81 \%$ de la muestra en cada caso. Es necesario señalar que, en este apartado, el 5,65\% comenta que, pese a que señalan un método concreto, no lo llevan a cabo de forma pura, sino que optan por una mezcla de metodologías. Era notorio observando los resultados de la encuesta, ya que los porcentajes de los análisis superan el $100 \%$. El motivo es porque en determinados casos señalaban varias opciones metodológicas.

Las respuestas emitidas confirman que el profesorado está influido, en sus planteamientos didácticos, por las metodologías que provienen de Centroeuropa. Es necesario señalar que en ningún caso han mencionado los métodos creados en nuestro país, y analizando las respuestas de los cuestionarios se puede confirmar que todos los desconocían. Además, los conocimientos de estos métodos los han adquirido, fundamentalmente, a través de cursos de postgrado y de habilitación en Educación Musical de la Consellería de Educación. Al respecto, las 80 respuestas emitidas referentes al curso de postgrado así lo manifiestan, lo que supone un $64,52 \%$. En la misma línea, 83 corresponden al curso impartido por la Consellería (el $66,94 \%)$ - en este punto, hay muchos que contestan por duplicado, puesto que le llaman al curso de la Consellería "curso de postgrado", por lo que señalaron las dos opciones, pese a ser un sólo curso. Bastantes menos docentes (el 26,61\%) declaran haberlos logrado en su formación inicial, y el $32,26 \%$ en la Escuela de Magisterio o Facultad de Ciencias de la Educación. Queda un $3,23 \%$ de profesionales que declaran haberlos obtenido en sus países de origen (Gráfico 7). Nuevamente, como podemos apreciar a través de los datos, la mayoría de las personas considera que una parte de su formación la han recibido en el curso de postgrado y otra parte en sus estudios iniciales o estudiando la carrera. 
Gráfico 7 - Adquisición de conocimientos

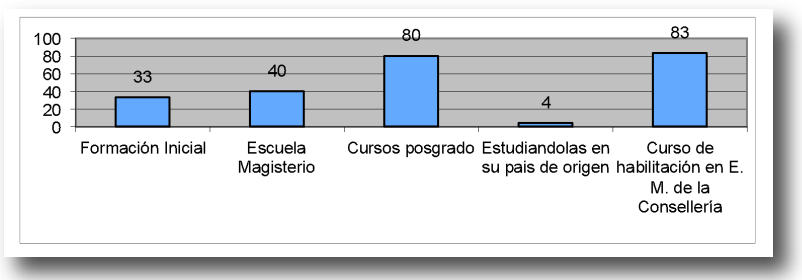

Para averiguar cuáles son los contenidos de la Educación Musical que más valoran en la Enseñanza Primaria, se solicitó al profesorado su estimación partiendo de once variables referidas a: percepción auditiva; lenguaje musical, agrupaciones instrumentales colectivas; estudios de un instrumento individual; folklore musical; enseñanza de canciones; movimiento; danzas; audiciones musicales; historia de la música; y "otras". A todas las variables les hemos adjudicado una valoración que va desde 1 como puntuación mínima hasta 6 como puntuación máxima. Los resultados obtenidos son bastante heterogéneos, como podemos apreciar en el gráfico 8. Aun así, en general, podemos concluir diciendo que la percepción auditiva es el dato más valorado para estos profesores de Música, ya que 78 le otorgan 6 puntos. En segundo lugar de importancia estaría la enseñanza de canciones, y le siguen las audiciones (aunque declaran no ser muchas las que utilizan). Le otorgan el cuarto lugar tanto al movimiento como a la enseñanza de nuestro folklore. En la opción "otras" no hemos recibido ninguna respuesta.

\section{Gráfico 8 - Aspectos que más valora en la enseñanza musical}

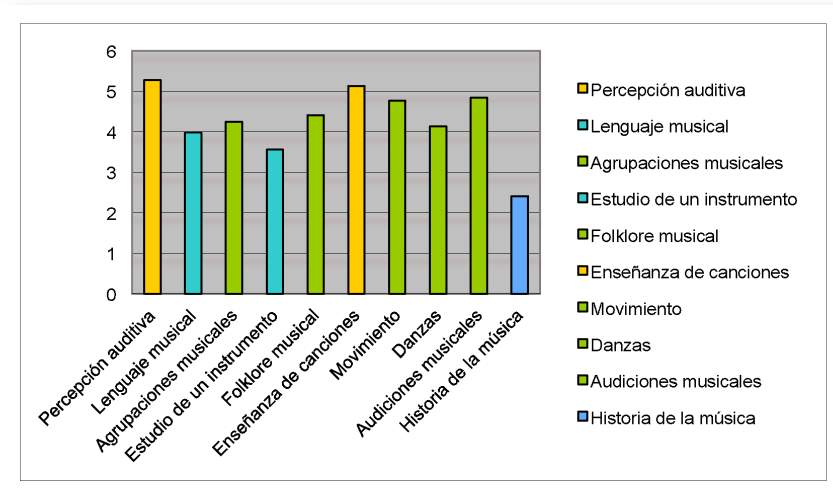




\section{Conclusiones}

En relación con los objetivos marcados en este estudio podemos decir que se ha comprobado que la formación inicial del profesorado gallego encargado de impartir Música en los centros de Educación Primaria tiene dos orígenes diferentes: el $91.13 \%$ procede de planes de estudio anteriores al 91 , por lo que no ha estudiado la especialidad de Educación Musical en la carrera de Magisterio, y tan solo el $8.87 \%$ restante procede de estudios específicos para formar profesores de esta disciplina.

En los antiguos planes de estudios (Plan 1950, 1967, 1971) la presencia de la Música era escasa, por lo que difícilmente su formación podía tener relevancia, limitándose a incluir, en el mejor de los casos, al aprendizaje de algunas canciones y el estudio de la flauta, relegando las danzas y otros instrumentos al olvido, entre otras cosas, porque no se trataba de formar profesores de Música, ya que esta especialidad no existía. Evidentemente, en la enseñanza-aprendizaje no se incluían los métodos de Pedagogía Musical, que como se ha mencionado anteriormente son tan importantes en la formación Musical del alumnado de Educación Primaria. Este es, por lo tanto, junto con la insuficiencia de contenidos musicales en los planes de estudios antiguos, uno de los aspectos fundamentales que diferencian al profesorado que ha cursado estudios específicos de Educación Musical con respecto a aquellos que no los han realizado.

El elevado número de docentes que procede de planes de estudios antiguos accedió a la Enseñanza Musical a través de sucintos cursos de postgrado organizados por la Xunta de Galicia debido a la necesidad de recolocar al profesorado suprimido impartiendo las nuevas especialidades LOGSE. En esta línea, Abreu \& Moura (2014) evidencian que muchos programas de formación de profesores fallan por dedicarse a los elementos que componen el trabajo docente de forma puntual y no articulada, sin considerar las condiciones objetivas de la realidad escolar en la que actúa cada profesor. A nuestro juicio, estos datos son muy significativos, puesto que existe una escasa representación de profesionales que han realizado estudios concretos y específicos para impartir este tipo de docencia, con el consiguiente detrimento que ello supone no solo en el aprendizaje musical, sino también en la formación integral del alumnado. 
En cuanto a la concepción metodológica, pese a que lo recomendable, según Brufal (2013) y Chao (2010), sería que el profesorado fundamentase su práctica docente en la utilización de los métodos expuestos anteriormente de forma combinada (Dalcroze, Orff, Kodaly, Ward, Martenot y Willems), esto no se produce. Como hemos visto a través de los resultados, las metodologías más empleadas por el profesorado (Orff y Kodály) provienen de Centroeuropa $y$ inducen al uso del folklore en el aula. No obstante, los profesores desconocen esta faceta tan elemental de dichos métodos, por lo que no los utilizan con esa finalidad y, a la vez que declaran emplear la metodología Orff, aseguran que solo recurren a ella para el uso de los instrumentos, lo que indica que identifican - y por tanto confunden - instrumental Orff con metodología. A su vez, el profesorado que se muestra partidario del método Kodály señala que sólo utiliza los signos fononímicos. En el resto de las metodologías no especifican como lo hacen, ni con qué finalidad, por lo que podemos claramente asegurar que poseen un conocimiento muy superficial de las mismas. Por otra parte, una amplia mayoría considera que el método empleado para su labor docente contribuye al desarrollo integral de su alumnado, si bien no son capaces de explicar por qué ni en qué medida les favorece.

En conclusión, siendo la Música una materia fundamental y básica en la enseñanza-aprendizaje, y teniendo en cuenta la ayuda que aporta al conocimiento, la motivación, la socialización, el rendimiento de los alumnos, no debería quedar en el olvido de los profesionales, de la administración y de los profesores a la hora de planificar el currículum, a la vez que su docencia debería estar en manos de personal específicamente cualificado. Coincidimos con Alves, Azevedo y Gonçalves (2014) cuando señalan que es necesario forjar el modo de apoyar el aprendizaje profesional y la construcción de la identidad profesional de los docentes al inicio de su carrera. Desde aquí nuestro compromiso para buscar las mejores maneras de dar un impulso para que nadie se vea privado de sus beneficios, y contribuir a que, al igual que en otros países, el profesorado encargado de su transmisión cuente con una formación adecuada. 


\section{Referencias}

Abreu, D., \& Moura, M. (2014). Construção de instrumentos teórico-metodológicos para captar a formação de professores. Educação e Pesquisa, 40(2), 401-414.

Alves, M. G., Azevedo, N. R., \& Gonçalves, T. (2014). Satisfação e situação profissional: Um estudo com professores nos primeiros anos de carreira. Educação e Pesquisa, 40(2), 365-382.

Bernabé, M. M. (2012). Importancia de la música como medio de comunicación intercultural en el proceso educativo. Teoría de la Educación - Revista Interuniversitaria, 24(2), 107-127.

Brufal, J. D. (2013). Los principales métodos activos de Educación Musical en Primaria: Diferentes enfoques, particularidades y directrices básicas para el trabajo en el aula. Artseduca, 5, 6-21.

Camacho, J. (2006). En torno a la formación inicial y permanente del profesorado. Avances en Supervisión Educativa - Revista de la Asociación de Inspectores de Educación de España, 3. Disponible en: http://www.adide.org/revista/index.php ?option=com_content\&task=view\&id=30\&ltemid=30.

Chao, R. (2010). La enseñanza del folklore gallego en los colegios de Educación Primaria de Galicia. Villalba: Conservatorio Profesional de Música.

Chao, R., Mato, M. D., \& Ferreiro, F. J. (2014). Music therapy in adolescent disruptive behavior. Procedia - Social and Behavioral Sciences, 132, 608-614.

Chao, R., Mato, M. D., \& Muñoz, J. M (2014). ¿Contribuyen los centros de Educación Primaria de Galicia a la conservación del folklore? Revista Lusófona de Educação [en prensa].

Climent, N., \& Carrillo, J. (2002). Una propuesta para la formación inicial de maestros. Ejemplificación: Los triángulos, una situación de primaria. Revista EMA Investigación e Innovación en Educación Matemática, 7(2), 171-205.

Climent, N., \& Carrillo, J. (2003). El dominio compartido de la investigación y el desarrollo profesional. Una experiencia en matemáticas con maestras. Enseñanza de las Ciencias, 21(3), 387-404.

Díaz, M. (2012). Investigamos, luego avanzamos. Revisa Electrónica d'Investigació Educativa y Socioeducativa, 2, 68-76.

Elliot, D. J. (2005). Praxial Music Education: Reflections and dialogues. Oxford: Oxford University Press.

Galvis, R. V. (2007). De un perfil docente tradicional a un perfil docente basado en competencias. Acción Pedagógica, 16, 48-57.

Imbernón, F. (2012). La investigación sobre y con el profesorado. La repercusión en la formación del profesorado, ¿cómo se investiga? Revista Electrónica de Investigación Educativa, 14(2), 1-9.

Levitin, D. (2011). Tu cerebro y la música. Barcelona: RBA.

Levitin, D. J., \& Álvarez, J. M. (2008). Tu cerebro y la música: El estudio científico de una obsesión humana. Barcelona: RBA.

López de la Calle, M. A. (2009). La formación de los maestros de Educación Infantil para la comprensión de la música y su uso didáctico en Galicia. REIFOP, 12(1), 107- 
120.

Marcelo, C. (2009). Profesorado principiante e inserción profesional a la enseñanza. Barcelona: Octaedro.

Marcelo, C. (2011). Evaluación del desarrollo profesional docente. A Coruña: Davinci.

Márquez, A. C. (2009). La formación inicial para el nuevo perfil del docente de Secundaria. Relación entre la teoría y la práctica (Tesis doctoral). Universidad de Málaga, Málaga.

Mourshed, M., Chijioke, C., \& Barber, M. (2010). How the world's most improved school systems keep getting better. McKinsey \& Company. Disponible en: http://mckinseyonsociety.com/downloads/reports/ Education/How-the-WorldsMost-Improved-School- Systems-Keep-Getting-Better_Downloadversion_Final.pdf.

Organización para la Cooperación y Desarrollo Económico [OCDE] (2012). PISA 2009 Technical Report. OECD Publishing.

Organización para la Cooperación y Desarrollo Económico [OCDE] (2013). PISA 2012 data base en www.pisa.oecd.org.

Pascual, P. (2006). Didáctica de la música en Educación Infantil. Madrid: Pearson Prentice Hall.

Pérez Aldeguer, S. \& Leganés Lavall, E. (2012). La música como herramienta interdisciplinar. Un análisis cuantitativo en el aula de lengua extranjera de primaria. Revista de Investigación en Educación, 10, 127-143.

Reyes, R., \& Pech, S. J. (2007). Preocupaciones de los profesores ante la reforma integral de la Educación Secundaria en México. REICE - Revista Electrónica Iberoamericana sobre Calidad, Eficacia y Cambio en Educación, 5(3), 173-189.

Toscano, C. M., \& Fonseca, M. C. (2012). La música como herramienta facilitadora del aprendizaje del inglés como lengua extranjera. Teoría de la Educación - Revista Interuniversitaria, 24(2), 197-213.

Touriñán, J. M., \& Longueira, S. (2010). La música como ámbito de educación Educación por la música y para la música. Teoría de la Educación - Revista Interuniversitaria, 22(2), 151-181.

Zabalza, M. A., \& Zabalza, M. A (2011). La formación del profesorado en Educación Infantil. Revista Participación Educativa - Tribuna Abierta, 16, 103-113. 


\section{A FORMAÇÃO MUSICAL DO PROFESSOR ESPECIALISTA NOS CEIP GALEGOS}

\section{Resumo}

A formação de professores é uma das preocupações dos países que lideram os resultados do relatório PISA, já que a qualificação dos docentes não confirma a sua eficácia como profissionais nem a qualidade do seu trabalho. A escassa formação musical dos alunos que acedem nos últimos anos a várias Faculdades de Educação da Galiza levou-nos a analisar as causas e, consequentemente, analisar a formação dos professores responsáveis pelo ensino de música nas escolas primárias (CEIP). Foi aplicado um questionário a uma amostra de 124 professores, dos quais 112 pertencem a escolas públicas e 12 a privadas. Os resultados podem ajudar os profissionais a melhorar e dirigir de forma diferente a sua ação educativa.

Palavras-chave

Formação de professores; Educação Musical; Ensino primário; Galiza 
THE MUSICAL TRAINING OF TEACHERS IN CEIPS IN GALICIA

\begin{abstract}
One of the main concerns of the countries that lead off PISA rankings is teachers' training, as someone's degree title cannot confirm their efficacy as a professional or a warranty of the quality of their work. The scarce musical training of students that have accessed different Schools of Education in Galicia during the past few years has lead us to analyze the causes and consequently to find out what is the training of the teachers in charge of the music subject in primary education schools (CEIP). We have made a questionnaire with a sample of 124 teachers, 112 of which teach in public centers, 12 in public-private. The results may help professionals to improve and approach differently their educational actions.
\end{abstract}

\title{
Keywords
}

Teachers' training; Musical education; Primary education; Galicia

Recebido em abril/2015 Aceite para publicação em julho/2015

i Departamento de Composición, Facultad de Ciencias de la Educación, Universidad de A Coruña, España.

ii Departamento de Pedagogía y Didáctica, Facultad de Ciencias de la Educación, Universidad de A Coruña, España.

iii Departamento de Ingeniería Mecánica y Diseño Industrial I, Escuela Superior de Ingeniería, Universidad de Cádiz, España.

Toda a correspondência relativa a este artigo deve ser enviada para: Rocío Chao Fernández, Facultad de Ciencias de la Educación, Universidad de A Coruña, Campus de Elviña S/N 10071, A Coruña. E-mail: rocio.chao@udc.es 